BRIGHT LINES OF $F c$ II IN THE SPECTRUM OF HD 50138

By O. Struve and P. Swings

This remarkable spectrum has been described by Merrill, ${ }^{1}$ who investigated the variation in the intensity of the bright violet component of $\mathrm{H} \beta$ and announced the presence of weak emission lines of forbidden $[O \mathrm{I}] 6300$ and 6363 . On his plates, extending from December 26, 1920, until December 13, 1929, the $F e$ II lines were observed only in absorption. A spectrogram taken at the McDonald Observatory on April 19, 1940, shows Fe Ir 4924, 5018,5169 to be weak, rather broad emission lines. The character of $F e$ II 4233 is uncertain. The widths of the $F e$ II emission lines are greater than those of the strong red component of $\mathrm{H} \beta$ and may be equal to the total widths of both $\mathrm{H} \beta$ components. We suspect a very weak emission line at $\lambda 6300$, but the plate is not good in this region. $\mathrm{CaK}$ is very sharp and must be of interstellar origin. The central absorption lines of $\mathrm{H}$ are very strong.

McDonald Observatory

Fort Davis, Texas

June 1940

\title{
APPOINTMENTS IN THE LICK OBSERVATORY
}

\section{By W. H. Wright}

At a recent meeting of the Board of Regents of the University of California, Dr. Frank E. Ross, of Pasadena, California, was appointed Alexander F. Morrison Research Associate in the Lick Observatory for the year 1940 -41. Dr. Ross's researches in photography, in mensurational problems of astronomy, and in lens design, are well known. In particular, Dr. Ross designed the photographic lens of the new Carnegie 20 -inch double astrographic camera of the Lick Observatory. The mounting for this instrument, which was built by the Warner \& Swasey Company, was recently installed in its new dome. It is expected that Dr. Ross, in his capacity as research associate, will be concerned primarily with the testing of the 20 -inch lens when the latter is completed, and with various related problems incident to the use of the telescope.

\footnotetext{
${ }^{1}$ Ap. J., 73, 348, 1931.
} 